\title{
The Importance of Being Variable
}

\author{
Douglas D. Garrett, ${ }^{1,2}$ Natasa Kovacevic, ${ }^{1}$ Anthony R. McIntosh, ${ }^{1,2}$ and Cheryl L. Grady ${ }^{1,2,3}$ \\ ${ }^{1}$ Rotman Research Institute, Baycrest, Toronto, Ontario M6A 2E1, Canada, ${ }^{2}$ Department of Psychology, University of Toronto, Toronto, Ontario M5S 3G3, \\ Canada, and ${ }^{3}$ Department of Psychiatry, University of Toronto, Toronto, Ontario M5T 1R8, Canada
}

New work suggests that blood oxygen level-dependent (BOLD) signal variability can be a much more powerful index of human age than mean activation, and that older brains are actually less variable than younger brains. However, little is known of how BOLD variability and task performance may relate. In the current study, we examined BOLD variability in relation to age, and reaction time speed and consistency in healthy younger (20-30 years) and older (56-85 years) adults on three cognitive tasks (perceptual matching, attentional cueing, and delayed match-to-sample). Results indicated that younger, faster, and more consistent performers exhibited significantly higher brain variability across tasks, and showed greater variability-based regional differentiation compared with older, poorerperforming adults. Also, when we compared brain variability- and typical mean-based effects, the respective spatial patterns were essentially orthogonal across brain measures, and any regions that did overlap were largely opposite in directionality of effect. These findings help establish the functional basis of BOLD variability, and further support the statistical and spatial differentiation between BOLD variability and BOLD mean. We thus argue that the precise nature of relations between aging, cognition, and brain function is underappreciated by using mean-based brain measures exclusively.

\section{Introduction}

Typical functional magnetic resonance imaging (fMRI) "activation" patterns result from analyses of averaged signals within voxel, within person. Although this approach has yielded great strides since the inception of fMRI, it remains that the brain's natural state is variable (Faisal et al., 2008; Raichle, 2010). Much work exists on the presence of variability and noise in the brain (Biswal et al., 1995; Stein et al., 2005; Faisal et al., 2008), but relatively few studies have considered brain variability as an individual differences measure of interest. Recent work indicates that blood oxygen level-dependent (BOLD) variability-based brain patterns are highly powerful indicators of age (Garrett et al., 2010a; Samanez-Larkin et al., 2010), nearly eliminate any useful age-predictive variance offered by the mean signal, and are virtually uncorrelated with age-related mean-based patterns (Garrett et al., 2010a). These findings suggest the presence of an effective variance-based "signal" within what many consider to be undesirable "noise" in fMRI.

Interestingly, few studies have examined the functional correlates of brain variability. Does it help or hinder us from respond-

\footnotetext{
Received Oct. 27, 2010; revised Jan. 20, 2011; accepted Jan. 24, 2011.

This work was supported by Canadian Institutes of Health Research grants to C.L.G. (Grant MOP14036) and A.R.M. (Grant MOP13026), and a JS MCDonnell Foundation grant to A.R.M. C.L.G. is supported also by the Canada Research Chairs program, the Ontario Research Fund, and the Canadian Foundation for Innovation. D.D.G. is supported by an IODE (Imperial Order Daughters of the Empire) War Memorial Award, a doctoral Canada Graduate Scholarship from the Natural Sciences and Engineering Research Council of Canada, the Sir James Lougheed Award of Distinction from Alberta Scholarship Programs, the Naomi Grigg Fellowship for Postgraduate Studies in Gerontology from Soroptimist International of Toronto, and the Men's Service Group Graduate Student Fellowship from the Rotman Research Institute, Baycrest. And, our apologies to Oscar Wilde, but the title of this paper wrote itself.

The authors declare no conflicts of interest.

Correspondence should be addressed to Douglas D. Garrett, Rotman Research Institute, Baycrest, 3560 Bathurst Street, Toronto, Ontario M6A 2E1, Canada. E-mail: d.garrett@utoronto.ca.

DOI:10.1523/JNEUROSCI.5641-10.2011

Copyright $\odot 2011$ the authors $\quad 0270-6474 / 11 / 314496-08 \$ 15.00 / 0$
}

ing faster or more consistently? Available studies suggest there are certain functional benefits [more accurate and less variable cognitive performance (McIntosh et al., 2008)] and detriments [poorer financial decision making (Samanez-Larkin et al., 2010)] of greater brain variability, depending on measure, population, and brain region. In the context of aging, much evidence supports the idea that older age yields various neural processing inefficiencies, which may manifest from degraded structural, functional, neuromodulatory, or genetic sources (Li et al., 2001; MacDonald et al., 2009). Importantly, these forms of neural inefficiency are argued not only to yield age-related slowing, but also age-related increases in response variability across a host of different cognitive tasks and samples (MacDonald et al., 2006a, 2009). Notably, although unexamined, one possible basis for typical age-related neural processing deficits and concomitant performance decrements is a critical lack of brain/variability. Our recent work suggests that at fixation, older brains are generally less variable than younger brains in a broad subset of regions (Garrett et al., 2010a). This reduced variability could reflect weakened functional connectivity (Fox et al., 2006; Nir et al., 2008), poorer neuronal signal detection ( $\mathrm{Li}$ et al., 2006), reduced dynamic range (Shew et al., 2009), or a limited ability to explore different network states (McIntosh et al., 2010). In any case, it remains unknown how BOLD variability and performance (i.e., response variability and speed) are related across different cognitive tasks with older age. Should age-related reductions (Garrett et al., 2010a) in brain variability also yield poorer performance on cognitive tasks spanning various domains, such results may reveal a novel and complementary source of age-related processing inefficiencies.

In the present study, we examined relations between brain signal variability and both chronological age and cognitive performance [mean reaction time (RT) and response variability] in 
young and older adults. Based on our previous work (McIntosh et al., 2008; Garrett et al., 2010a), we anticipated that greater brain variability would be associated with younger age and better performance overall, and that variability- and mean-based brain patterns would prove largely nonoverlapping.

\section{Materials and Methods \\ Sample}

Our sample consisted of 18 young adults (mean age $=25.79 \pm 3.28$ years, range 22-31 years, 10 women) and 27 older adults (mean age $=66.46 \pm$ 8.25 years, range $56-85$ years, 14 women). Most participants were right handed (three in each group were left handed), and all were screened using a detailed health questionnaire to exclude health problems and/or medications that might affect cognitive function and brain activity, including strokes and cardiovascular disease. Structural MRIs also were inspected to rule out severe white matter (WM) changes or other abnormalities. There was no relation between age and performance on the mini-mental state examination (Folstein et al., 1975). The present experiment was approved by the Research Ethics Board at Baycrest, and all participants gave informed consent for their participation (following the guidelines of the Research Ethics Board at Baycrest and the University of Toronto) and were paid for their participation.

\section{Cognitive tasks}

All brain analyses were performed using volumes acquired during task blocks from a block design study (each task block was preceded and succeeded by a 20-s-long fixation block) (Grady et al., 2010). Visual stimuli were bandpass filtered white noise patches with different center frequencies. For the purposes of the present study, we analyzed withinperson reaction time means and variability scores across three cognitive tasks: (1) perceptual matching (PMT); (2) attentional cueing (ATT); and (3) delayed match-to-sample (DMS). For PMT a sample stimulus appeared centrally in the upper portion of the screen along with three choice stimuli located in the lower part of the screen (for $4000 \mathrm{~ms}$ ). The task was to indicate which of the three choice stimuli matched the sample. Six such trials occurred in each PMT block (eight blocks total $=48$ trials). For ATT, a stimulus appeared for $1500 \mathrm{~ms}$ in the center of the upper part of the screen. Then an arrow pointing either to the right or to the left appeared (in the lower part of the screen) with the sample stimulus for $1500 \mathrm{~ms}$. The arrow was removed and $500 \mathrm{~ms}$ later, two stimuli appeared in the right and left locations for $3000 \mathrm{~ms}$. The task was to attend only to the location that had been cued by the arrow, and press one of two buttons to indicate whether or not the cued target stimulus matched the sample. There were four trials in each ATT block ( 8 blocks total $=32$ trials). Finally, in the DMS task, a sample stimulus was presented for $1500 \mathrm{~ms}$ in the center of the upper portion of the screen followed by a delay of $2500 \mathrm{~ms}$ (blank screen). Then, three choice stimuli were presented for $3000 \mathrm{~ms}$ in the lower portion of the screen and the participants had to press one of three buttons to indicate which of the three stimuli matched the previously seen sample. There were four trials in each DMS block ( 8 block total $=32$ trials). In all tasks, the intertrial interval was $2000 \mathrm{~ms}$ (see Notes).

Before scanning, participants were tested in a mock scanner to determine within-subject accuracy thresholds for each task. These thresholds indicated the difference in center frequency between stimuli necessary for accuracy in each task to be $\sim 80 \%$ during the scanning session. This criterion helped equate task difficulty across participants before performing tasks in the scanner.

\section{MRI scanning and preprocessing}

We acquired images with a Siemens Trio $3 \mathrm{~T}$ magnet. We first obtained a $\mathrm{T} 1$ weighted anatomical volume using spoiled gradient-recalled acquisition in a steady state [echo time $(\mathrm{TE})=2.6 \mathrm{~ms}$, retention time $(\mathrm{TR})=$ $2000 \mathrm{~ms}$, field of view $(\mathrm{FOV})=256 \mathrm{~mm}$, slice thickness $=1 \mathrm{~mm}$ ) for coregistration with the functional images and to ensure that there were no significant brain abnormalities in any participants. T2* functional images $\left(\mathrm{TE}=30 \mathrm{~ms}, \mathrm{TR}=2000 \mathrm{~ms}\right.$, flip angle $\left.=70^{\circ}, \mathrm{FOV}=200 \mathrm{~mm}\right)$ were obtained using echoplanar imaging acquisition. Each functional sequence consisted of $285-\mathrm{mm}$-thick axial slices, positioned to image the whole brain. A total of 144 volumes were collected for PMT and DMS, and 136 volumes for ATT. Images were registered to a nonlinear group average anatomical image (Kovacević et al., 2005; Chen et al., 2006; Levine et al., 2008) intended to serve as an unbiased anatomical template. We have used this template in previous studies of both young and older adults (for complete details on template construction, see Garrett et al., 2010a). Functional data were slice-time corrected using AFNI (http:// afni.nimh.nih.gov/afni) and motion corrected using AIR (http://bishopw. loni.ucla.edu/AIR5/) by registering all functional volumes to the 100th volume within run. By averaging all functional volumes within a motioncorrected run, we calculated mean functional volumes. For each run, mean functional volume was registered with each subject's structural volume using a rigid body transformation model. After appropriate transform concatenations, from initial volume to the 100th volume within run, from mean run volume to structural volume, and from structural volume into our template space, we obtained a direct nonlinear transform from each initial fMRI volume into template space. We then applied the FMRIB Software Library (FSL)/FNIRT (FSL Non-linear Image Registration Tool) registration algorithm to find a nonlinear transform between our template and Montreal Neurological Institute (MNI) 152_T1 provided with FSL software (www. fmrib.ox.ac.uk/fsl). Data were smoothed using an $8 \mathrm{~mm}$ Gaussian kernel.

We performed several additional preprocessing steps intended to reduce data artifacts. We corrected functional volumes in the Common Template space via Independent Component Analysis within separate runs, as implemented in FSL/MELODIC (Multivariate Exploratory Linear Optimized Decomposition into Independent Components) (Beckmann and Smith, 2004). We further adjusted voxel time series by regressing out motion correction parameters, and WM and CSF time series. For WM and CSF regression, we extracted time series from unsmoothed data within small regions of interest (ROIs) in the corpus callosum and ventricles of the Common Template. ROIs were selected such that they were deep within each structure of interest (corpus callosum and ventricles) to avoid signal contamination from external tissues due to misregistration. Our rationale for using small ROIs and unsmoothed data was to ensure that the ROIs would not contain signal of interest (i.e., gray matter signal) for any subject. The choice of a one 4 $\mathrm{mm}^{3}$ voxel within the corpus callosum for WM and a same size voxel within one lateral ventricle for CSF was based on our experience of having excellent registration for these structures across ages. With a large age span in our data, it would be easy to introduce age-related bias if larger ROIs or smoothed data were used. Spatial smoothing mixes signals from neighboring voxels on one hand, and registration errors during spatial normalization on the other; both factors can contaminate WM and CSF time series due to the close proximity of gray matter voxels. And, although we used nonlinear registration to adjust for age-related differences in anatomy (i.e., atrophy), differences likely remained such that, e.g., larger CSF ROIs would have residual small, yet biased, age-dependent contributions from GM signal. These potential issues justified our use of only small ROIs and unsmoothed data during WM and CSF regression.

To localize regions from our functional output, we submitted MNI coordinates to the Anatomy Toolbox in SPM8, which applies probabilistic algorithms to determine the cytoarchitectonic labeling of MNI coordinates (Eickhoff et al., 2005, 2007). Regions not labeled using this method were located manually using the Atlas of the Human Brain (Mai et al., 2008) after transforming MNI coordinates to Talairach space with the Nonlinear Yale MNI to Talairach Conversion Algorithm (Lacadie et al., 2008) and associated online Java-based applet.

\section{Data analyses}

Behavioral measures. To prepare the data for examining each of our cognitive tasks, we adopted an approach used in several previous studies (Hultsch et al., 2000, 2008; MacDonald et al., 2006b; Dixon et al., 2007). We set a lower bound ( $150 \mathrm{~ms}$ ) for legitimate responses for each task on the basis of minimal RTs suggested by prior research (MacDonald et al., 2006b; Dixon et al., 2007). We then trimmed extreme outliers relative to the rest of the sample on each task ( $\geq 4000 \mathrm{~ms}$ ). We established final upper bounds for each task by dropping all trials $>3$ SDs from withinperson means. The number of trials dropped across all participants and tasks was negligible (179/5040 total trials). For each task, we then im- 
puted missing values for outlier trials by using regression imputation (as implemented in SPSS 18.0).

Using these imputed values, we calculated mean RTs and intraindividual SDs of RTs (hereto referred to as mean $\mathrm{RT}_{\mathrm{T}}$ and $\mathrm{ISD}_{\mathrm{RT}}$, respectively) for each participant on each task according to previous recommendations (see below) (Hultsch et al., 2000, 2008; MacDonald et al., 2006b; Dixon et al., 2007). Computing $\mathrm{ISD}_{\mathrm{RTs}}$ on raw RTs can be problematic for many cognitive measures. Significant relations between various variables (e.g., age and possible practice effects) and mean ${ }_{\mathrm{RTs}}$ are typically observed, and mean ${ }_{\mathrm{RTs}}$ are often positively correlated with raw SD values. Thus, for example, age differences in response variability could simply be due to preexisting differences in $\operatorname{mean}_{\mathrm{RT}}$ (Hale et al., 1988), thus revealing little about the true state of variability. To disentangle these potential confounds from legitimate response variability, we used regression to residualize the effects of age, block, trial and all their interactions from all RT trials separately for each task. Further, in light of a recent suggestion to evaluate the linearity assumption used in the residualization of RT data when calculating intraindividual variability (Schmiedek et al., 2009), we also examined the possible presence of nonlinearities between RT trials and age, block, and trial number. We found none when examining up to a quartic trend. Further, our subsequent linear-only model runs revealed no effects of block, trial, or any related interactions on RTs. However, a significant effect of age was noted for each task, thus justifying the residualization of age from the data (PMT, $R^{2}=0.30, p<$ 0.0001 ; ATT, $R^{2}=0.16, p<0.0001$; DMS, $\left.R^{2}=0.15, p<0.0001\right)$. We then computed standardized RT residuals from this age-only model, followed by within-person $\mathrm{ISD}_{\mathrm{RTs}}$ on these residuals. As evidence for the lack of effects of block and trial, we found that correlations between residuals from our first linear model (including age, block, trial, and all interactions) and our second linear model (age only) were statistically redundant (all $r$ values $>0.99$ for all tasks).

Calculation of BOLD signal mean and SD. To calculate mean signal $\left(\operatorname{mean}_{\mathrm{BOLD}}\right)$ for each experimental condition, we first expressed each signal value as a percentage change from its respective block onset value, and then calculated a mean percentage change within each block and averaged across all blocks for a given condition. To calculate BOLD SDs $\left(\mathrm{SD}_{\mathrm{BOLD}}\right)$, we first normalized all condition blocks such that the overall $4 \mathrm{D}$ mean across brain and block was 100 to correct for possible lowfrequency artifacts for each condition. For each voxel, we then subtracted the block mean and concatenated across all blocks for each condition (for further details, see Garrett et al., 2010a). Finally, we calculated voxel SDs across this concatenated mean-block corrected time series. Our previous work demonstrated that our preprocessing pipeline and brain variability calculations reduced the average voxel SD by $50 \%$ across the brain, yet the predictivity of $\mathrm{SD}_{\mathrm{BOLD}}$ more than doubled in magnitude (Garrett et al., 2010a). This suggests that the further removal of possible "junk noise" sources hones, rather than reduces the impact of, the $\mathrm{SD}_{\mathrm{BOLD}}$ signal.

Other recent work on temporal brain variability (Samanez-Larkin et al., 2010) used an alternative measure of variability [the mean squared successive difference (MSSD)] (von Neumann et al., 1941) intended to prevent mean shifts in the data from overestimating true dispersion in the data. Others (Mohr and Nagel, 2010) have commented that our use of the $\mathrm{SD}_{\mathrm{BOLD}}$ in neuroimaging (Garrett et al., 2010a) may be questionable when task data are examined because we have not controlled for mean shifts that could potentially overestimate true signal variability. On the contrary (Garrett et al., 2010b), our measure of $\mathrm{SD}_{\mathrm{BOLD}}$ is remarkably similar to the MSSD because we remove block means before $\mathrm{SD}_{\mathrm{BOLD}}$ computation; the MSSD is intended to detrend the data, and this is exactly what is achieved when block normalizing before $\mathrm{SD}_{\mathrm{BOLD}}$ calculation (see Garrett et al., 2010a, their Fig. 2). In the present study, we calculated the correlation between our $\mathrm{SD}_{\mathrm{BOLD}}$ measure (calculated as noted above) and the MSSD (without block normalization) at fixation only and across the entire time series of fixation and task blocks. We computed this within and across subjects, and within and across runs. Unsurprisingly, in all cases, our $\mathrm{SD}_{\mathrm{BOLD}}$ measure and the MSSD were statistically redundant (all $r$ values $>0.97$ ).

Partial least squares analysis of relations between brain and age/behavior. For each of the two brain measures ( $\mathrm{SD}_{\mathrm{BOLD}}$ and mean $\mathrm{BOLD}_{\mathrm{D}}$ ), we performed separate partial least squares (PLS) analyses (behavioral PLS)
(McIntosh et al., 1996), which allow the identification of multivariate patterns of relations between brain and age/behavior relations. This type of analysis begins with the correlation matrix between our three variables of interest (age, mean $_{\mathrm{RT}}$, and $\mathrm{ISD}_{\mathrm{RT}}$ ) and each voxel's signal on each task; correlations are calculated across subjects. The correlation matrix is decomposed using singular value decomposition to produce latent variables, consisting of the correlation strength on one hand (i.e., the singular value), and so-called "brain saliences" on the other (i.e., a weighting pattern across brain voxels that optimally expresses the correlation). In the present study, because we examined age, mean $_{\mathrm{RT}}$, and $\mathrm{ISD}_{\mathrm{RT}}$ for PMT, ATT, and DMS, nine latent dimensions were possible for each PLS analysis. We then calculated so-called "brain scores" (akin to component scores in principal component analyses) by taking the dot product of the brain saliences and a given subject's brain measures. Thus, in a single measure, a brain score indicates the degree to which a subject expresses the multivariate spatial pattern captured by a given age- and behaviordriven latent variable. Significance of detected relations between multivariate spatial patterns and age/behavior was assessed using 1000 permutation tests of the singular value associated with each latent variable. A subsequent bootstrapping procedure revealed the robustness of voxel saliences across 1000 bootstrapped resamples of our data. By dividing each voxel's bootstrap mean salience by its estimated SE, we obtained so called "bootstrap ratios" as normalized estimates of robustness. We thresholded bootstrap ratios at a value of 3.00, which approximates a 99\% confidence interval.

\section{Results}

Separately for $\mathrm{SD}_{\mathrm{BOLD}}$ and mean $\mathrm{BOLD}_{\mathrm{B}}$ analyses, we examined age, mean $_{\mathrm{RTs}}$, and $\mathrm{ISD}_{\mathrm{RTs}}$ in the same model, given known positive relations among these three measures in the aging literature (Hultsch et al., 2008). In our data, these relations were robust and positive, regardless of cognitive task (see Notes).

\section{Relations between BOLD variability and age, mean $_{\mathrm{RTs}}$, and ISD $_{\mathrm{RTs}}$}

First, we used behavioral multivariate PLS (McIntosh et al., 1996) to calculate the presence and strength of age- and behavioraldependent multivariate spatial patterns of $\mathrm{SD}_{\mathrm{BOLD}}$ across all three cognitive tasks. We found a very strong relation between age, behavior, and brain variability within a single, significant latent variable (singular value $=69.04$, permuted $p<0.0001$ ). Bootstrapped estimated confidence intervals around correlations between age and performance and the multivariate spatial pattern demonstrated similar effects across age, behavior, and tasks (Fig. $1 a)$. The pattern of voxels demonstrating this relation between $\mathrm{SD}_{\mathrm{BOLD}}$ and age and behavior effects was widely distributed and is shown in Figure $2 a$. The vast majority of regions ( $84 \%$ of all robust voxels) exhibited greater variability with younger age, faster mean $_{\mathrm{RTs}}$, and lower $\mathrm{ISD}_{\mathrm{RTs}}$ (e.g., occipital cortex, cingulate cortex, angular gyrus, shown in blue). Several small clusters (16\% of all robust voxels) showed the opposite pattern, in which lesser variability occurred with younger age, faster mean $_{\mathrm{RTs}}$, and lower $\mathrm{ISD}_{\mathrm{RTs}}$ (e.g., cerebellum, right anterior prefrontal cortex, shown in yellow/red) (see Notes).

Relations between mean ${ }_{\mathrm{BOLD}}$ and age, mean ${ }_{\mathrm{RTs}}$, and $\mathrm{ISD}_{\mathrm{RTs}}$

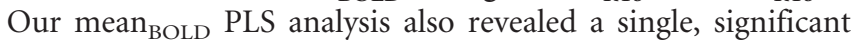
latent variable (singular value $=53.37$, permuted $p<0.0001$ ). Like for $\mathrm{SD}_{\mathrm{BOLD}}$, bootstrapped confidence intervals demonstrated similar effects across age, behavior, and tasks; however, the magnitudes of these correlations were somewhat lower (Fig. $1 b$ ). The resulting multivariate spatial pattern (Fig. $2 b$ ) indicated that less mean activity in a host of regions (e.g., occipital cortex, middle frontal gyri, and left anterior cingulate; shown in yellow/ red) correlated with younger age, faster mean $_{\mathrm{RTs}}$, and lower 

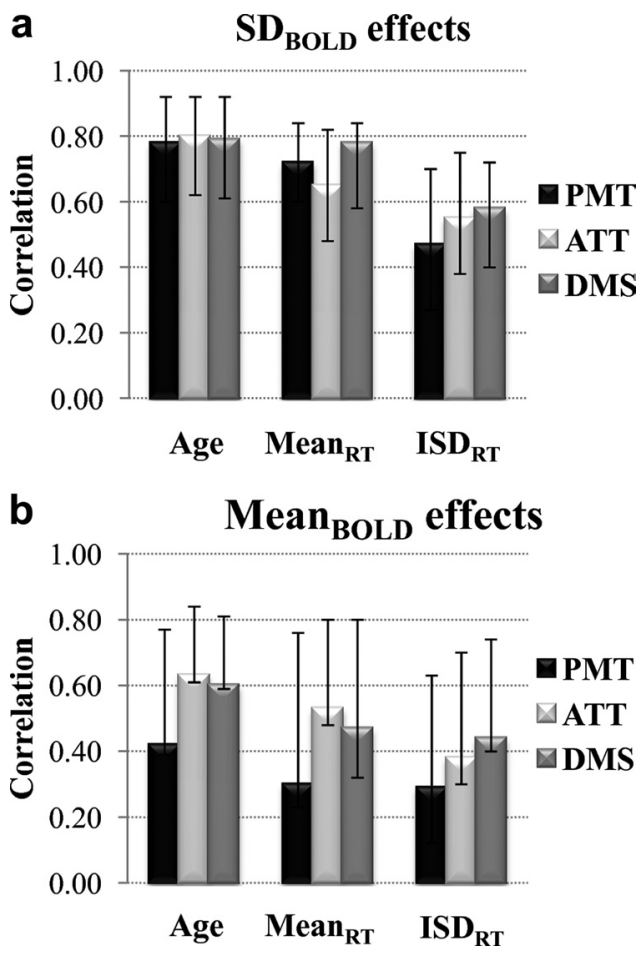

Figure 1. $\quad \boldsymbol{a}, \boldsymbol{b}$, Correlations (Pearson $r$ ) between age, mean ${ }_{R T}$, and $I S D_{R T}$ across tasks and $\mathrm{SD}_{\text {BOLD }}(\boldsymbol{a})$ and mean ${ }_{\text {BOLD }}(\boldsymbol{b})$. Both model effects resulted from the first latent variable from separate task PLS model runs (one for $\mathrm{SD}_{\text {BOLD }}$ and one for mean ${ }_{B O L D}$ ). Error bars represent bootstrapped $95 \%$ confidence intervals. Although our sample did not consist of middle-aged adults, analyzing age as either a continuous or dichotomous variable (young vs old) made little difference in our PLS model runs. $R^{2}$ values were within $1.4 \%$ across relations involving brain, age, and performance, with no differences in permuted $p$-values. Thus, we elected to maintain the use of age as a continuous measure, just as we did to evaluate initial bivariate relations (see Notes).

$\mathrm{ISD}_{\mathrm{RTs}}$; only one small cluster exhibited greater mean activity (i.e., precentral gyrus; shown in blue) (see Notes).

How do mean ${ }_{\mathrm{BOLD}}$ and $\mathrm{SD}_{\mathrm{BOLD}}$ spatial patterns compare?

From Figure 2, $a$ and $b$, it is obvious that our $\mathrm{SD}_{\mathrm{BOLD}}$ and mean $_{\text {BOLD }}$ spatial patterns have far more differences than similarities. Convergent with our previous findings (Garrett et al., 2010a), this suggests that the two brain measures (SD and mean) revealed different topographical manifestations of the same age and behavioral effects across tasks. Image overlay plots using only robust bootstrapped voxels (Fig. $2 c, d$ ) highlighted similarities and differences between these spatial patterns. Notably, there were few similarities between brain measures outside of occipital cortex, and even within this region, the majority of overlapping voxels have opposite directionality (Fig. $2 d$, shown in red). Thus, younger age, and faster and more consistent RTs were associated with greater brain variability, but less mean activity in these few overlapping regions.

To quantify the degree of spatial overlap on a whole-brain level, we correlated mean ${ }_{\mathrm{BOLD}}$ and $\mathrm{SD}_{\mathrm{BOLD}}$ PLS parameter estimates across all voxels (not just those voxels that surpass a bootstrap threshold of 3.00 or more). We subsequently computed a bootstrapped $95 \%$ confidence interval around this correlation. Similar to our previous work (Garrett et al., 2010a), there was no evidence for global spatial overlap $(r=-0.02$; bootstrap confidence interval $=-0.29,0.23$ ). Because we computed a correlation across all voxels that may show either positive, negative, or no correlation across brain measures (which could effectively cancel each other out if there is no clear directional pattern across voxels), it is possible that some degree of spatial overlap, as seen in Figure $2 d$, will not guarantee correlation across the brain. In our data, this was exactly the case. For example, negative correlations between $\mathrm{SD}_{\mathrm{BOLD}}$ and mean $\mathrm{BOLD}_{\mathrm{BD}}$ measures in occipital cortex were counteracted by $\mathrm{SD}_{\mathrm{BOLD}}$ clusters in the cerebellum that were

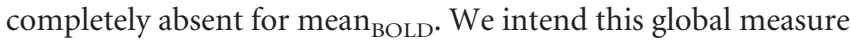
to indicate whether one could expect to know something about brain variance by knowing something about mean brain activity; in our data, the answer is largely no. Even when we calculated correlations between $\mathrm{SD}_{\mathrm{BOLD}}$ and mean $\mathrm{BOLD}_{\mathrm{B}}$ values across only robust voxels, the correlation remained low $(r=-0.24)$. As work on brain variability continues, specific types of spatial overlap between mean ${ }_{\mathrm{BOLD}}$ and $\mathrm{SD}_{\mathrm{BOLD}}$ maps may be expected; at present however, we find little evidence for meaningful overlap.

\section{Direction and magnitudes of brain variability by age and performance}

Our $\mathrm{SD}_{\mathrm{BOLD}}$ analyses revealed a relative pattern in which more brain variability largely corresponded to younger age and better performance (Fig. $2 a$, blue regions), whereas only a few areas showed a correspondence between more brain variability, older age and poorer performance (Fig. $2 a$, yellow/red regions; referred to here as yellow regions). In light of these results, we then asked, precisely how different are levels of variability across regions (blue and yellow), both within and across age and performance levels (i.e., in terms of actual, rather than relative, average $\mathrm{SD}_{\mathrm{BOLD}}$ differences)? There are two possible patterns of brain variability levels that could produce the age- and performance-based effects we found in the current study: (1) younger, better-performing adults exhibit a similar brain variability level across both blue and yellow regions (lesser variability-based regional brain differentiation) in Figure $2 a$, while older, poorer-performing adults exhibit lower brain variability in blue than in yellow regions (greater regional differentiation), or; (2) younger, better performers present with higher brain variability levels in blue regions than in yellow (greater regional differentiation), and older adults exhibit similar levels of brain variability across blue and yellow regions (lesser regional differentiation). To examine this, we first took all robust voxels from our $\mathrm{SD}_{\text {BOLD }}$ analysis (those that surpassed a BSR threshold of \pm 3.00 ) and separated them into those from blue regions and those from yellow regions. We then calculated the across-task average within-person SD for all blue voxels and all yellow voxels, separately. We calculated within-person voxel SDs across tasks because of the remarkable similarity of our PLS results across tasks (Fig. 1a, see the overlap in confidence intervals). Finally, using mixed modeling, we compared a group of young, faster, and more behaviorally consistent participants (22-31 years of age, $>1.00 \mathrm{SD}$ below the sample $\mathrm{Mean}_{\mathrm{RT}}$ across tasks and the sample ISD $\mathrm{RT}_{\mathrm{RT}}$ across tasks; $n=13$ ) to older, slower, less consistent participants $(56-85$ years, $>1.00 \mathrm{SD}$ above the sample Mean ${ }_{\mathrm{RT}}$ across tasks and $\mathrm{ISD}_{\mathrm{RT}}$ across tasks; $n=20$ ) on blue and yellow $\mathrm{SD}_{\mathrm{BOLD}}$. We found that younger, faster, and more consistent participants had $78 \%$ more brain variability in blue regions than they had in yellow regions, whereas older, slower, and more inconsistent participants had essentially the same level of brain variability across blue and yellow regions (group $\times$ region interaction, $F_{(1,31)}=165.44, p<0.0001$, partial $\eta^{2}=0.84$; Fig. 3). Further, younger and better-performing participants had $37 \%$ more brain variability in blue regions, and $27 \%$ less brain variability in yellow regions compared with the older, poorer-performing group. These results suggest that older and poorer-performing adults have a vastly reduced range of brain 
a

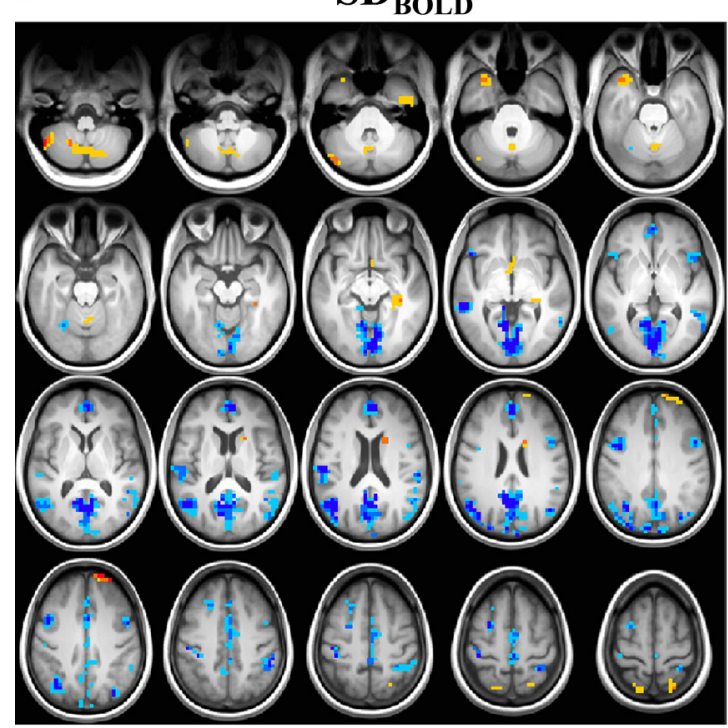

C

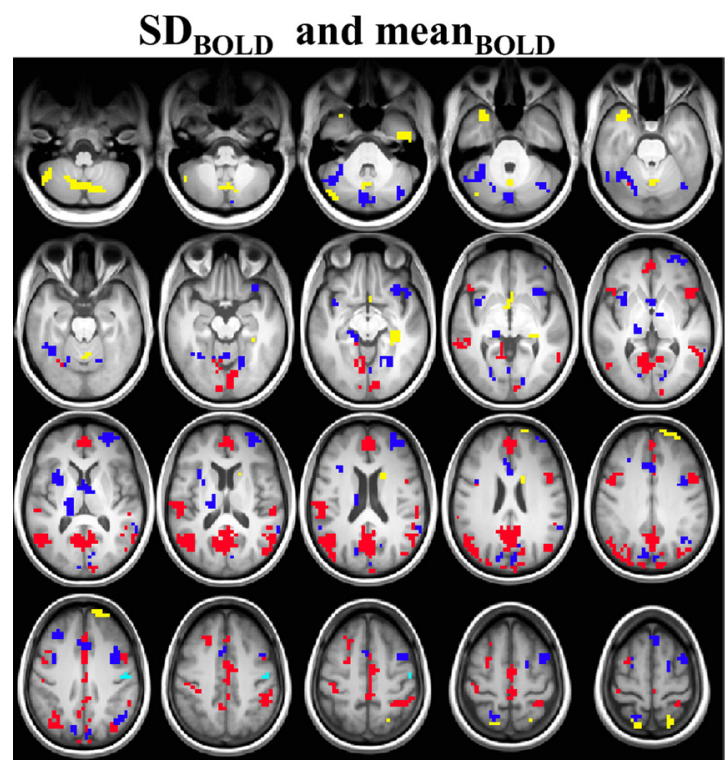

b

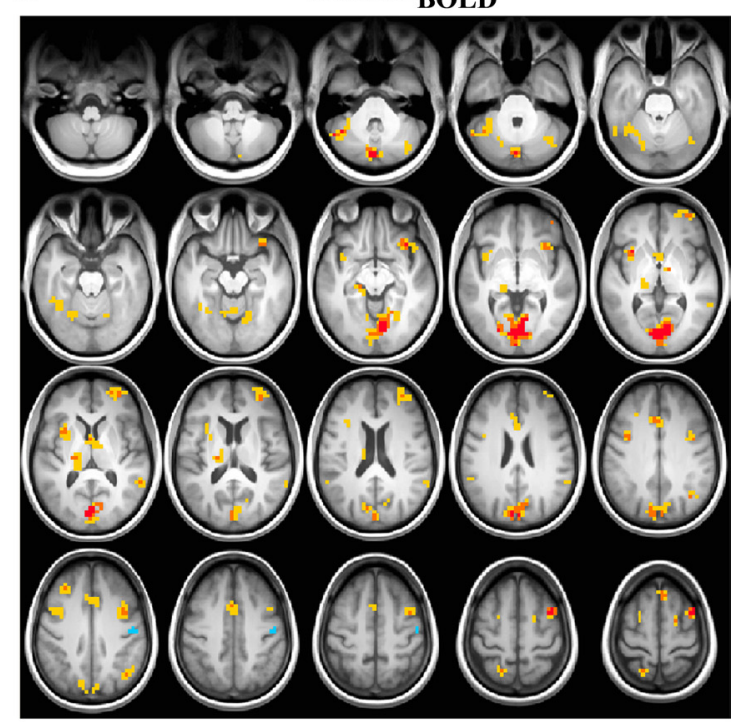

d

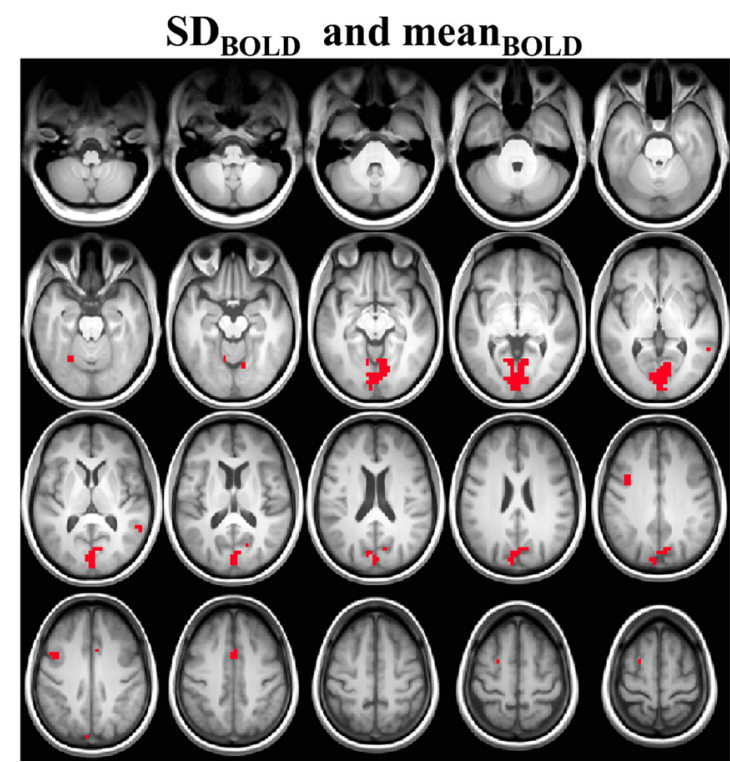

Figure 2. PLS brain patterns and overlay plots. $\boldsymbol{a}$, Blue regions indicate greater and yellow/red regions indicate lesser brain variability with younger age, and faster and more consistent RT performance. $\boldsymbol{b}$, Blue regions indicate greater and yellow/red regions indicate lesser mean brain activity with younger age, and faster and more consistent RT performance. In both $\boldsymbol{a}$ and $\boldsymbol{b}$, all robust areas surpassed a thresholded bootstrap ratio (salience/SE) of $\geq 3.00$ (for yellow/red regions) or $\leq-3.00$ (for blue regions). Darker colors indicate greater robustness. $c$, 0 verlay plot highlighting differences between $\mathrm{SD}_{B O L D}$ and mean ${ }_{B O L D} S$ satial patterns. Red, Greater $\mathrm{SD}_{B O L D}$ with younger age and better performance, but no mean ${ }_{B O L D}$ effect; yellow, lesser $\mathrm{SD}_{B O L D}$ with younger age and better performance, but no mean ${ }_{B O L D}$ effect; cyan, greater mean ${ }_{B O L D}$ with younger age and better performance, but no $\mathrm{SD}_{B O L D}$ effect; blue, lesser mean ${ }_{B O L D}$ with younger age and better performance, but no $S D_{B O L D}$ effect. $\boldsymbol{d}$, Overlay plot highlighting overlap between $\mathrm{SD}_{B O L D}$ and mean ${ }_{B O L D}$ Spatial patterns. Red, Greater $\mathrm{SD}_{B O L D}$ and lesser mean ${ }_{B O L D}$ with younger age and better performance. All images represent every other slice in $z$-direction.

variability across robust voxels (and thus, reduced variabilitybased regional differentiation) compared to younger and betterperforming adults.

\section{Discussion}

Our results confirmed that greater brain variability across a broad set of regions was strongly associated with younger age, faster response times, and less variable RTs (a small number of voxel clusters exhibited the opposite effect). For mean ${ }_{\text {BOLD }}$, a significant age and performance effect also was found, although spatial patterns were essentially orthogonal across brain measures, and any regions that did overlap were largely opposite in directionality of effect. Our work continues to support the idea that assessing
BOLD variability reveals a highly distinct and powerful brain pattern not captured by mean signal, either at fixation (Garrett et al., 2010a) or on task.

A more variable brain is a more effective brain

The directionality of our $\mathrm{SD}_{\mathrm{BOLD}}$ effect suggests that overall, greater brain variability is functional, and our results converge with previous work. McIntosh et al. (2008) found that greater EEG signal variability correlated with more consistent reaction times and more accurate performance, and may index a more sophisticated neural system that can explore multiple functional states. We have argued that, as a nonlinear dynamical system, the brain functions at the "edge of criticality" between any number of 


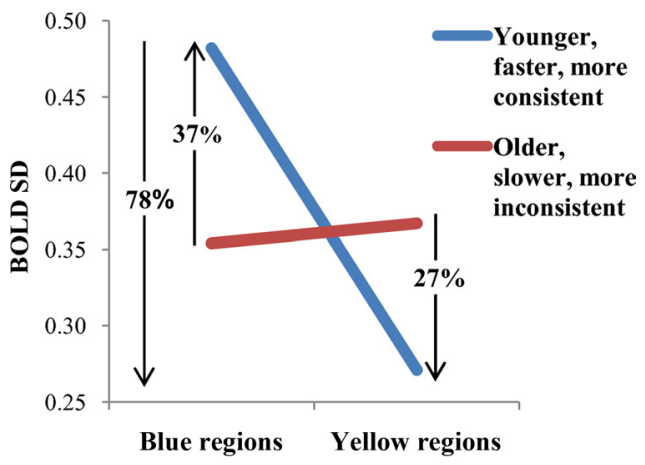

Figure 3. Levels of brain variability in robust blue and yellow regions from our $\mathrm{SD}_{B O L D} P L S$ analysis. Blue and yellow regions refer to those in Figure $2 a$ (we label yellow/red in Fig. $2 a$ as yellow here). Fast and slow refer to $\leq 1$ and $\geq 1$ SD from the sample mean ${ }_{R T}$ across tasks; consistent and inconsistent refer to $\leq 1$ and $\geq 1$ SD from the sample average $\mid S D_{R T}$ across tasks. Blue areas represent $84 \%$ and yellow areas represent $16 \%$ of robust brain voxels identified in the PLS SD ${ }_{B O L D}$ analysis. Thus, the group difference noted here for blue regions is much more prominent in brain than is the difference noted for yellow regions. Unsurprisingly, a separate analysis of overall brain variability (i.e., $\mathrm{SD}_{\mathrm{BOLD}}$ of all robust voxels, regardless of whether blue or yellow) also revealed a strong group difference, $F_{(1,31)}=21.77, p<0.0001$, partial $\eta^{2}=$ 0.41 ; younger, faster, more consistent adults exhibited $25 \%$ more brain variability than older, slower, more inconsistent adults across tasks.

possible states or functional network configurations (McIntosh et al., 2010; Deco et al., 2011). When variability is too low, there is little capacity for the system to explore these states, yielding the potential for the system to remain in a single state. With relatively greater variability, and upon fluctuations in variability, the brain is more capable of transitioning from one state to another. Stochastic resonance research suggests that there is an optimal level of noise that facilitates neural detection of weak incoming signals, and too little or too much noise results in less efficient information processing (Li et al., 2006; McDonnell and Abbott, 2009). Relatedly, balanced neural excitation and inhibition (that innately yields a given amount of variability) also aids the brain in responding to the greatest range of stimuli (i.e., maximal dynamic range); during hypoexcitable (less variable) and hyperexcitable (too variable) states, dynamic range, and thus neural efficiency, suffer (Shew et al., 2009). In our study, because participants with greater brain variability were younger and more efficient processors of information, these participants' variability patterns may represent an "optimal" state characterized by a greater ability to transition between brain states and by increased dynamic range. Conversely, it would appear that older, slower, more behaviorally inconsistent participants lack a critical amount of brain variability (at least in the blue areas noted in Fig. 2a) for optimal neural function.

Some investigators (Ma et al., 2006; Beck et al., 2008) have argued that variability is essential for the nervous system to operate in an optimal, probabilistic, Bayesian manner; that is, neural variability yields adaptability across levels of stimulus uncertainty in one's environment by integrating available information and subsequently computing probabilities for the most efficient response. Optimal, probabilistic neural function may require relatively greater task-driven brain variability to improve one's ability to learn and to handle ongoing external environmental demands. For example, recent work with monkeys suggests that single-cell variability increases during visuomotor learning, thus facilitating the exploration of possible motor states and better adaptation to new environments (Mandelblat-Cerf et al., 2009). In our study, if younger, faster, more consistent participants represent an optimally functioning group, then greater brain variability may serve as a proxy measure of their probabilistic, Bayesian-based neural system in action. Further, if it is true that variability provides the kinetic energy for networks to explore possible functional architectures (McIntosh et al., 2010; Deco et al., 2011), then by being in a constant state of exploration, it is plausible that the brain continually generates predictions about the most optimal network configuration for a given input.

However, it is certainly not always the case that relatively greater brain variability is functional. Although the number of regions that exhibited lower variability in younger, faster, and more behaviorally consistent participants were far fewer in number (accounting for only 16\% of robust voxels), they are noteworthy. Interestingly these regions predominated in the cerebellum and subcortical structures (e.g., right caudate nucleus, right hippocampus), both here and in our previous study examining age differences in brain variability during fixation (Garrett et al., 2010a). Similarly, using a financial decision task, a recent study (Samanez-Larkin et al., 2010) also noted greater age-related signal variability primarily in a number of subcortical regions (e.g., right caudate nucleus, right thalamus). The authors also found that higher variability in the nucleus accumbens (NAcc) correlated with riskier financial decisions, and that this NAcc-performance relation mediated the effect between age and performance. Surprisingly though, the authors found no regions that exhibited greater variability with younger age or better performance, whereas our largest pockets of $\mathrm{SD}_{\mathrm{BOLD}}$ activation were strongly associated with younger age and better performance (e.g., occipital cortex, much of the cingulate cortex). Although our respective studies suggest some convergence at the subcortical level, future research should address differences between subcortical and cortical brain variability effects, directionality of effects, and task types used.

With regard to actual levels of brain variability, our results indicated that younger, better-performing brains had 78\% more variability in blue regions than they did in yellow regions, whereas older, poorer-performing brains maintained the same level of variability across regions. This suggests the presence of dramatic between-subject differences in across-region differentiation in $\mathrm{SD}_{\mathrm{BOLD}}$. Thus, although we found that overall, a more variable brain is an effective brain, the brain's ability to maintain greater signal stability in certain regions (in our case, in yellow regions in Fig. $2 a$ ) is also a critical marker of both younger age and better task performance. Importantly, the lack of brain variability differences across regions in the older and poorer-performing group may offer novel evidence for brain dedifferentiation with age. Dedifferentiation theory (Baltes and Lindenberger, 1997; Park and Reuter-Lorenz, 2009; Park et al., 2010) suggests that older brains may become less functionally distinct during task performance (i.e., through reductions in the selectivity and specificity of neural processes). Our results indicate age-based dedifferentiation of signal variability across a variety of regions, thus representing an alternative and informative view of cortical selectivity.

\section{A notable inversion between behavioral and brain variability, and relations with age}

Over the last several years, interest in behavioral variability has heightened, particularly with regard to response variability in reaction time studies. Response variability typifies older adults, as well as a host of other age- and non-age-related conditions (Hultsch et al., 2008; MacDonald et al., 2009). This variability may index reductions in nervous system integrity, and could result from various sources of neural inefficiency that impede consistent information transmission within the nervous system (MacDonald et al., 2009). Notably, we found that more consis- 
tent response patterns (as well as younger age and faster RT), correlated with greater brain variability; it thus appears that a lack of BOLD variability in many regions could serve as a novel index of inefficient processing in this context. There are several reasons the directionality of this effect could exist. For example, some forms of brain variability appear to be a direct function of coherence and connectivity between brain regions (Fox et al., 2006; Nir et al., 2008). Along with increased response variability (MacDonald et al., 2006a, 2009), older adults also consistently exhibit reduced or altered brain connectivity at both structural (Sullivan and Pfefferbaum, 2006) and functional levels (Andrews-Hanna et al., 2007; Grady et al., 2010), possibly then, resulting in reduced brain variability. If greater brain variability indeed indicates a more sophisticated, complex neural system (McIntosh et al., 2008), then given age-related reductions in connectivity, it is unsurprising that older, more inconsistently performing adults would show lower brain variability than younger, more consistently performing adults. Interestingly, evidence exists for a U-shaped curve representing the development of behavioral variability (i.e., that young children show the greatest variability, young adults the least variability, and older adults increase in variability into late life) (Williams et al., 2005). Combined with McIntosh et al.'s (2008) results that young adults have more variable brains and less variable RT performance than younger children, our current results may provide evidence for an inverted form of this developmental U-shaped curve with regard to brain variability (in which older adults have less variability than young adults). Future research on BOLD variability across the lifespan would clarify this curve's existence and form.

\section{Complementarities and other future directions}

The present results provide unique and potentially complementary results to a bourgeoning field of brain variability research (McIntosh et al., 2010). Intriguingly, recent alternative approaches applied to young adults (Xue et al., 2010) demonstrate that spatial variability (i.e., spatial pattern dissimilarity across stimuli repetitions, in which spatial patterns are determined using mean BOLD values at each voxel) is associated with poorer memory performance. Future research could thus examine the hypothesis that temporal and spatial pattern variability may be inversely associated. Further, the present work complements existing research demonstrating links between heightened behavioral variability and degraded structural, functional, neuromodulatory, and genetic sources (Li et al., 2001; MacDonald et al., 2009). The future study of plausible positive relations between the integrity of such sources and temporal BOLD variability would help greatly to establish the underlying substrate necessary for temporal brain variability to occur. Also, given the sensitivity of brain variability for detecting age (Garrett et al., 2010a) and performance, the potential for brain variability to provide a useful marker in other age (e.g., dementia, mild cognitive impairment, stroke)- and non-age-related contexts (e.g., traumatic brain injury; schizophrenia) may be great.

\section{Conclusion}

In line with our previous findings (Garrett et al., 2010a), our $\mathrm{SD}_{\text {BOLD }}$ pattern in the current study was statistically robust and spatially differentiated from mean activation. Overall, younger, faster, and more consistent participants exhibited higher brain variability across tasks, providing evidence that BOLD variability indeed plays an important functional role. Younger, better performers also exhibited greater regional $\mathrm{SD}_{\mathrm{BOLD}}$-based differentiation compared with older, poorer-performing adults. We suggest researchers continue to compare and contrast BOLD variability and BOLD mean in future research. In the ongoing study of relations between the brain and important phenomena of interest (behavior, cognition, development, disease), it is probable that the true state of those relations are underappreciated by using mean BOLD $_{\mathrm{B}}$ measures exclusively.

\section{Notes}

Supplemental material is posted at http://douglasdgarrett.com/ publications and includes Figure S1 (visual depiction of tasks), Table S1 ( $r$ matrix of age and performance relations), and Tables $\mathrm{S} 2$ and $\mathrm{S} 3$ (peaks, $\mathrm{MNI}$ coordinates, bootstrap ratios, and cluster sizes for $\mathrm{SD}_{\mathrm{BOLD}}$ and mean $_{\mathrm{BOLD}}$ results, respectively). This material has not been peer reviewed.

\section{References}

Andrews-Hanna JR, Snyder AZ, Vincent JL, Lustig C, Head D, Raichle ME, Buckner RL (2007) Disruption of large-scale brain systems in advanced aging. Neuron 56:924-935.

Baltes PB, Lindenberger U (1997) Emergence of a powerful connection between sensory and cognitive functions across the adult life span: a new window to the study of cognitive aging? Psychol Aging 12:12-21.

Beck JM, Ma WJ, Kiani R, Hanks T, Churchland AK, Roitman J, Shadlen MN, Latham PE, Pouget A (2008) Probabilistic population codes for Bayesian decision making. Neuron 60:1142-1152.

Beckmann CF, Smith SM (2004) Probabilistic independent component analysis for functional magnetic resonance imaging. IEEE Trans Med Imaging 23:137-152.

Biswal B, Yetkin FZ, Haughton VM, Hyde JS (1995) Functional connectivity in the motor cortex of resting human brain using echo-planar MRI. Magn Reson Med 34:537-541.

Chen XJ, Kovacevic N, Lobaugh NJ, Sled JG, Henkelman RM, Henderson JT (2006) Neuroanatomical differences between mouse strains as shown by high-resolution 3D MRI. Neuroimage 29:99-105.

Deco G, Jirsa VK, McIntosh AR (2011) Emerging concepts for the dynamical organization of resting state activity in the brain. Nat Rev Neurosci 12:43-56.

Dixon RA, Garrett DD, Lentz TL, MacDonald SW, Strauss E, Hultsch DF (2007) Neurocognitive markers of cognitive impairment: Exploring the roles of speed and inconsistency. Neuropsychology 21:381-399.

Eickhoff SB, Stephan KE, Mohlberg H, Grefkes C, Fink GR, Amunts K, Zilles K (2005) A new SPM toolbox for combining probabilistic cytoarchitectonic maps and functional imaging data. Neuroimage 25:1325-1335.

Eickhoff SB, Paus T, Caspers S, Grosbras MH, Evans AC, Zilles K, Amunts K (2007) Assignment of functional activations to probabilistic cytoarchitectonic areas revisited. Neuroimage 36:511-521.

Faisal AA, Selen LP, Wolpert DM (2008) Noise in the nervous system. Nat Rev Neurosci 9:292-303.

Folstein MF, Folstein SE, McHugh PR (1975) “Mini-mental state.” A practical method for grading the cognitive state of patients for the clinician. J Psychiatr Res 12:189-198.

Fox MD, Snyder AZ, Zacks JM, Raichle ME (2006) Coherent spontaneous activity accounts for trial-to-trial variability in human evoked brain responses. Nat Neurosci 9:23-25.

Garrett DD, Kovacevic N, McIntosh AR, Grady CL (2010a) Blood oxygen level-dependent signal variability is more than just noise. J Neurosci 30:4914-4921.

Garrett DD, Kovacevic N, McIntosh AR, Grady CL (2010b) All withinsubject BOLD SDs are not created equal [electronic response to Mohr PN and Nagel IE, Variability in brain activity as an individual difference measure in neuroscience? J Neurosci 30:7755-7757.] http://www.jneurosci.org/ content/30/23/7755.full/reply jneuro_el_68849.

Grady CL, Protzner AB, Kovacevic N, Strother SC, Afshin-Pour B, Wojtowicz M, Anderson JA, Churchill N, McIntosh AR (2010) A multivariate analysis of age-related differences in default mode and task-positive networks across multiple cognitive domains. Cereb Cortex 20:1432-1447.

Hale S, Myerson J, Smith GA, Poon LW (1988) Age, variability, and speed: between-subjects diversity. Psychol Aging 3:407-410.

Hultsch DF, MacDonald SW, Hunter MA, Levy-Bencheton J, Strauss E (2000) Intraindividual variability in cognitive performance in older adults: comparison of adults with mild dementia, adults with arthritis, and healthy adults. Neuropsychology 14:588-598. 
Hultsch DF, Strauss E, Hunter MA, MacDonald SWS (2008) Intraindividual variability, cognition, and aging. In: The handbook of aging and cognition (Craik FI, Salthouse TA, eds), pp 491-556. New York: Psychology Press.

Kovacević N, Henderson JT, Chan E, Lifshitz N, Bishop J, Evans AC, Henkelman RM, Chen XJ (2005) A three-dimensional MRI atlas of the mouse brain with estimates of the average and variability. Cereb Cortex 15:639-645.

Lacadie CM, Fulbright RK, Rajeevan N, Constable RT, Papademetris X (2008) More accurate Talairach coordinates for neuroimaging using non-linear registration. Neuroimage 42:717-725.

Levine B, Kovacevic N, Nica EI, Cheung G, Gao F, Schwartz ML, Black SE (2008) The Toronto traumatic brain injury study: injury severity and quantified MRI. Neurology 70:771-778.

Li SC, Lindenberger U, Sikström S (2001) Aging cognition: from neuromodulation to representation. Trends Cogn Sci 5:479-486.

Li SC, Van Oertzen T, Lindenberger U (2006) A neurocomputational model of stochastic resonance and aging. Neurocomputing 69:1553-1560.

Ma WJ, Beck JM, Latham PE, Pouget A (2006) Bayesian inference with probabilistic population codes. Nat Neurosci 9:1432-1438.

MacDonald SW, Nyberg L, Bäckman L (2006a) Intra-individual variability in behavior: links to brain structure, neurotransmission and neuronal activity. Trends Neurosci 29:474-480.

MacDonald SW, Hultsch DF, Bunce D (2006b) Intraindividual variability in vigilance performance: does degrading visual stimuli mimic age-related “neural noise?" J Clin Exp Neuropsychol 28:655-675.

MacDonald SW, Li SC, Backman L (2009) Neural underpinnings of withinperson variability in cognitive functioning. Psychol Aging 24:792-808.

Mai JK, Paxinos G, Voss T (2008) Atlas of the human brain, Ed 3. New York: Elsevier.

Mandelblat-CerfY, Paz R, Vaadia E (2009) Trial-to-trial variability of single cells in motor cortices is dynamically modified during visuomotor adaptation. J Neurosci 29:15053-15062.

McDonnell MD, Abbott D (2009) What is stochastic resonance? Definitions, misconceptions, debates, and its relevance to biology. PLoS Comput Biol 5:1-9.

McIntosh AR, Bookstein FL, Haxby JV, Grady CL (1996) Spatial pattern analysis of functional brain images using partial least squares. Neuroimage 3:143-157.
McIntosh AR, Kovacevic N, Itier RJ (2008) Increased brain signal variability accompanies lower behavioral variability in development. PLoS Comput Biol 4:e1000106.

McIntosh AR, Kovacevic N, Lippe S, Garrett D, Grady C, Jirsa V (2010) The development of a noisy brain. Arch Ital Biol 148:323-337.

Mohr PN, Nagel IE (2010) Variability in brain activity as an individual difference measure in neuroscience? J Neurosci 30:7755-7757.

Nir Y, Mukamel R, Dinstein I, Privman E, Harel M, Fisch L, Gelbard-Sagiv H, Kipervasser S, Andelman F, Neufeld MY, Kramer U, Arieli A, Fried I, Malach R (2008) Interhemispheric correlations of slow spontaneous neuronal fluctuations revealed in human sensory cortex. Nat Neurosci 11:1100-1108.

Park DC, Reuter-Lorenz P (2009) The adaptive brain: Aging and neurocognitive scaffolding. Annu Rev Psychol 60:173-196.

Park J, Carp J, Hebrank A, Park DC, Polk TA (2010) Neural specificity predicts fluid processing ability in older adults. J Neurosci 30:9253-9259.

Raichle ME (2010) Two views of brain function. Trends Cogn Sci 14:180-190.

Samanez-Larkin GR, Kuhnen CM, Yoo DJ, Knutson B (2010) Variability in nucleus accumbens activity mediates age-related suboptimal financial risk taking. J Neurosci 30:1426-1434.

Schmiedek F, Lövdén M, Lindenberger U (2009) On the relation of mean reaction time and intraindividual reaction time variability. Psychol Aging 24:841-857.

Shew WL, Yang H, Petermann T, Roy R, Plenz D (2009) Neuronal avalanches imply maximum dynamic range in cortical networks at criticality. J Neurosci 29:15595-15600.

Stein RB, Gossen ER, Jones KE (2005) Neuronal variability: noise or part of the signal? Nat Rev Neurosci 6:389-397.

Sullivan EV, Pfefferbaum A (2006) Diffusion tensor imaging and aging. Neurosci Biobehav Rev 30:749-761.

von Neumann J, Kent RH, Bellinson HR, Hart BI (1941) The mean square successive difference. Ann Math Stat 12:153-162.

Williams BR, Hultsch DF, Strauss EH, Hunter MA, Tannock R (2005) Inconsistency in reaction time across the life span. Neuropsychology 19:88-96.

Xue G, Dong Q, Chen C, Lu Z, Mumford JA, Poldrack RA (2010) Greater neural pattern similarity across repetitions is associated with better memory. Science 330:97-101. 